\title{
Epidemiologia e estratégias de prevenção do câncer de pênis no estado do Maranhão
}

\author{
Epidemiology and strategies for the prevention of penile cancer in the state of Maranhão
}

Gissela Santos Lindosoํㅜ, Ercínia Gomes Duailibe Barros ${ }^{1}$, Lise Feitosa Novais Miranda ${ }^{1}$, Bruno Carvalho Campelo ${ }^{1}$, Karol Cristina Fonseca Moura ${ }^{1}$, Leandro de Assis Freitas ${ }^{2}$, Giovanna Valeria Belo Cordeiro ${ }^{*}$.

\begin{abstract}
Resumo: Dentre os diversos tipos de câncer e suas mais variadas formas está o câncer de pênis (CP), com níveis de incidência mais elevados em países em desenvolvimento. O CP é uma neoplasia rara, cujo tratamento, muitas vezes mutilante, causa efeitos devastadores aos pacientes. Logo, o objetivo desse estudo foi avaliar por meio de uma revisão sistemática o perfil epidemiológico do CP no Brasil e as principais estratégias de prevenção. Foi realizada uma prospecção de fontes tradicionais de conhecimento (como livros), artigos de periódicos eletrônicos obtidos do PUBMED/Medline, Scielo, CAPES e Lilacs publicados até 2018. Foram utilizados os descritores: câncer de pênis, epidemiologia e prevenção, sendo encontrados 23 artigos que atendiam aos critérios de inclusão. Normalmente o CP ocorre associado à fimose, tabagismo, DSTs, hábitos inadequados de higiene e resistência masculina em procurar assistência médica. Portanto, o câncer de pênis é uma doença de grande relevância para a saúde pública, uma vez que há um crescimento vertiginoso dos casos, que podem ser minimizados através de campanha de conscientização masculina.
\end{abstract}

Palavras-chave: Câncer de Pênis. Epidemiologia. Estratégias de Prevenção.

\begin{abstract}
Among the various types of cancer and its most varied forms, penile cancer (PC) is presented, with higher levels of incidence in developing countries. PC is a rare neoplasm, whose often mutilating treatment causes devastating effects on patients. This study aimed to evaluate, through a sitematic review, the epidemiological profile of PC in the State of Maranhao and the main prevention strategies. To do this, he carried out a survey of traditional sources of knowledge (such as books), articles from electronic journals taken from PUBMED / Medline, Scielo, CAPES and Lilacs published until 2018. There were used as descriptors: penile cancer; epidemiology and prevention, and was found 23 articles that corresponded the inclusion criteria. PC usually occurs associated with phimosis, smoking, STDs, poor hygiene habits, and male resistance to seeking medical attention. Therefore, PC is a disease of great relevance since there is a dizzying growth of cases which can be minimized through the male awareness campaign.
\end{abstract}

Keywords: Penile Cancer. Epidemiology. Prevention Strategies.

\footnotetext{
${ }^{1}$ Universidade Ceuma, São Luís - MA, Brasil; ${ }^{2}$ Hospital Beneficência Portuguesa, São Paulo- SP, Brasil;

${ }^{*}$ Rua Josué Montello, oㅜ 1, Renascença II, São Luís, Maranhão, Brasil. CEP: 65075-120. (98) 999733373. E-mail: giovannabelo@terra.com.br
} 


\section{Introdução}

O carcinoma de pênis é raro e manifesta-se por lesões e mudanças de coloração, pelo aparecimento de úlcera persistente ou tumor situado na glande, no prepúcio ou no corpo do pênis e nos gânglios inguinais. A neoplasia de pênis é um grave problema de saúde pública por ser uma doença agressiva e causar lesões mutilantes, podendo levar à perda do órgão e afetando a auto-estima e a vida sexual e afetiva do doente ${ }^{1}$.

O carcinoma de células escamosas é responsável por $95 \%$ dos casos de neoplasias malignas do pênis. Acomete, em geral homens entre a quinta e a sexta décadas de vida. Segundo Pompeo ${ }^{2} 0$ carcinoma escamoso, tipo mais comum, é classificado quanto à histologia em três categorias, a saber: bem diferenciado, equivalente a $70 \%$ a $80 \%$ dos casos, moderadamente diferenciado e indiferenciado. Há estudos que estabelecem sua relação com a infecção pelo HPV (human papillomavirus papiloma vírus humano), que é uma doença sexualmente transmissível.

De acordo com um estudo realizado por Barnholtz-Sloan et al. ${ }^{3}$ a incidência de câncer do pênis nos Estados Unidos entre 1973 e 2002 foi de 0,69 por 100.000 habitantes. O Brasil é um dos países com um dos maiores índices de registro de câncer de pênis no mundo, com frequência variável dependendo da região estudada. O Instituto Nacional do Câncer estimou mais de 4600 casos de câncer de pênis no Brasil em 2013, sendo a região Nordeste a mais prevalente ${ }^{3,6}$. Segundo o Instituto Nacional do Câncer ${ }^{5}$, o Maranhão no ano de 2015 apresentava $1,13 \%$ dos casos de câncer de pênis no Nordeste, esse número cresceu para $1,25 \%$ em 2016.

Assim, para se obter uma diminuição na ocorrência de câncer de pênis, é de extrema importância uma maior atenção a saúde do homem com pesquisas relacionadas às causas e fatores de risco desta patologia, além de fornecer informações à população sobre medidas de prevenção da doença. O objetivo deste estudo foi avaliar, por meio de revisão da literatura, o perfil epidemiológico do câncer de pênis na população maranhense e também as principais estratégias de prevenção.

\section{Material e Método}

Esse trabalho foi realizado a partir de uma revisão de literatura, que apresentou como subsídio a consulta de livros acadêmicos, artigos de periódicos eletrônicos retirados do PUBMED/Medline, Scielo, CAPES e Lilacs. Foram adotados os seguintes critérios para seleção dos artigos: todas as categorias de artigo (original, revisão de literatura, atualização, relato de caso etc.); artigos com resumos e textos completos disponíveis para análise; aqueles publicados no idioma português até 0 ano de 2018 e artigos que apresentassem em seus títulos e/ou resumos os seguintes descritores em ciências da saúde (DeCS): câncer de pênis, epidemiologia e prevenção.

Do material obtido, resultando em 37 artigos, procedeu-se a leitura minuciosa de cada um, destacando aqueles que responderam ao objetivo proposto por este estudo. Seguindo os critérios de inclusão, 23 artigos foram selecionados para análise, os quais são referenciados no presente texto.

\section{Revisão de literatura}

\section{Câncer de pênis}

Segundo o Instituto Nacional do Câncer ${ }^{4,6}$, o câncer é um dos problemas de saúde pública mais complexos que o sistema de saúde brasileiro enfrenta, dada a sua extensão epidemiológica, social e econômica. Observa-se ainda que, em média, um terço dos casos novos de câncer no mundo podem ser prevenidos.

$O$ câncer pode surgir em qualquer parte do corpo, sendo que alguns tipos 
são classificados de acordo com a localização primária do tumor, tais como colo do útero, mama e pulmão. O número de casos novos de câncer cresce a cada ano 4,6 .

Dentre os tipos de neoplasias que acometem o sexo masculino, destaca-se - carcinoma de pênis, que embora acometa pequena parcela da população, está associado à alta morbidade decorrente da própria doença e/ou de seu tratamento, provocando altos impactos psicológicos nos pacientes ${ }^{5}$.

As principais causas envolvidas na etiologia são a falta de higiene, presença de fimose ou excesso de prepúcio e associação com o HPV em 30 a $50 \%$ dos casos. Os sintomas mais frequentes são ferimentos que não cicatrizam mesmo após tratamento, caroços que não desaparecem e que têm secreção e mau cheiro, vermelhidão ou coceira constante na glande de portadores de fimose e surgimento de tumores no pênis ou na região inguinal ${ }^{4,6}$.

\section{Epidemiologia}

O carcinoma de pênis não é uma doença frequente nos países desenvolvidos, correspondendo de $0,4 \%$ a $3 \%$ de todas as neoplasias malignas. 0 aumento da incidência ocorre a partir da sexta década de vida, sendo infrequente em adultos abaixo de 30 anos, e raramente descrito em crianças. Para países em desenvolvimento é problema importante de saúde, pois em algumas regiões da África, Ásia e América do Sul chega a corresponder até a $10 \%$ das neoplasias malignas no homem ${ }^{4,6}$.

As estimativas do Instituto Nacional de Câncer ${ }^{4,6}$ para 2010 não apontavam o CP como um dos mais frequentes no país, pois representa $2,1 \%$ de todos os tipos de câncer entre homens. Entretanto, sua ocorrência é $15 \%$ mais alta no Norte e no Nordeste, onde existem condições socioeconômicas mais precárias, com incidência de 1,3 a 2,7 por 100 mil habitantes. Em 2006 e 2007, a Sociedade Brasileira de Urologia (SBU), ao realizar um estudo epidemiológico, identificou 283 novos casos de CP no Brasil - destes, $53,02 \%$ ocorreram no Norte e no Nordeste, sendo o Maranhão (Gráfico 1), de maior destaque; em segundo lugar se destacou a região Sudeste, com $45,54 \%$. A maioria dos pacientes $(78 \%)$ tinha mais de 46 anos, e $7,41 \%$ menos de 35 anos $^{4,6}$.

Uma provável explicação para a alta incidência de câncer peniano no estado

Tabela 1. Comparação da incidência do câncer peniano entre alguns estados do Brasil.

\begin{tabular}{|l|c|c|c|}
\hline \multicolumn{1}{|c|}{ Estado } & $\begin{array}{c}\text { Números de } \\
\text { casos }\end{array}$ & Período & $\begin{array}{c}\text { Taxa de incidência } \\
(100.000 \text { homens/ano })\end{array}$ \\
\hline Maranhão & 392 & $2004-2014$ & 1,18 \\
\hline Bahia & 378 & $1997-2007$ & 0,72 \\
\hline Pará & 208 & $1996-2006$ & 0.46 \\
\hline Pernambuco & 88 & $2007-2012$ & 0,34 \\
\hline Rio de Janeiro & 230 & $2002-2008$ & 0,43 \\
\hline
\end{tabular}


do Maranhão é a alta taxa de infecção pelo HPV. Um estudo prospectivo não publicado (com 57 casos de câncer de pênis do Maranhão) relatou uma taxa de infecção pelo HPV de mais de $75 \%$. Esses dados são corroborados pelo fato de que - Maranhão tem a maior incidência de câncer do colo do útero no Brasil, cuja patogênese está diretamente relacionada à infecção pelo HPV ${ }^{1}$.

As características epidemiológicas e clínicas dos pacientes deste estudo foram semelhantes às relatadas em outros estudos, particularmente em regiões em desenvolvimento cuja realidade socioeconômica é semelhante a do Maranhão. O estado do Maranhão possui um Índice de Desenvolvimento Humano (IDH) de 0,639, considerado o menor do Brasil. Segundo o IBGE, $71,7 \%$ das famílias no Maranhão ganham menos de US \$220,00 por mês. Neste estudo, $71 \%$ dos pacientes relataram ser agricultores e $82,1 \%$ eram de áreas rurais. Quando comparados com outros estudos, os pacientes desse Estado apresentam taxas de tabagismo bastante baixas na população geral. Isso seria consistente com as taxas relativamente baixas de fumantes no estado do Maranhão ${ }^{9}$.

Embora a idade média de 58,5 anos seja semelhante à maioria dos estudos relatados na literatura, as séries brasileiras mostram uma maior frequência de casos em indivíduos jovens. Este estudo mostrou que $19,7 \%$ dos pacientes diagnosticados com câncer de pênis tinham $\leq 40$ anos de idade. Em jovens com carcinoma de células escamosas, o padrão de crescimento tumoral infiltrativo perineural, invasão e recorrência são mais prováveis. No entanto, a literatura e os indicadores prognósticos atuais não incluem a idade do diagnóstico como importante marcador prognóstico ${ }^{9}$.

\section{Manifestações Clínicas}

Geralmente o câncer de pênis iniciase como uma lesão na glande, que, ao não ser tratada, estende-se para 0 prepúcio e se infiltra nos tecidos adjacentes, como o tecido subepitelial conjuntivo, o corpo esponjoso e o corpo cavernoso, até invadir órgãos próximos como próstata e bexiga, levando à amputação e à danos mais extensos ${ }^{6}$.

Os padrões morfológicos de crescimento e o desenvolvimento do carcinoma epidermoide de pênis apresenta-se em quatro padrões principais: crescimento superficial, crescimento vertical, verrucoso e multicêntricos. O "crescimento superficial" ocorre quando o tumor é plano, de padrão epidermóide pouco invasivo, e corresponde a $1 / 3$ dos casos; 0 "crescimento vertical" é uma grande lesão ulcerada com padrão de crescimento sólido, agressivo e invasivo, ocorrendo em $20 \%$ dos pacientes; o "verrucoso" é lento, com três padrões histológicos: verrucoso propriamente dito, papilar e condilomatoso, que apresentam baixo índice de metástases; os "multicêntricos" são definidos por duas ou mais lesões separadas por tecido benigno. São tumores raros, sendo mais comuns no prepúcio, e podem estar associados a líquen escleroso ${ }^{9}$.

A queixa do paciente com câncer de pênis é comumente relacionada à presença de lesão vegetante ou de áreas de ulceração peniana. As lesões variam quanto às dimensões e com frequência o paciente procura o atendimento médico tardiamente por falta de recursos locais, conforme a Figura 1, ou mesmo por temer o tratamento cirúrgico ${ }^{9}$. A intensidade e localização das manifestações clínicas servem de base para o estadiamento do câncer de pênis, sendo utilizados dois sistemas: a classificação de Jackson e o sistema (TNM) ${ }^{6,7}$.

Jackson (1966) propôs um sistema de classificação baseado em critérios anatômicos, ganhando destaque entre as demais classificações pela sua praticidade. Atualmente, o sistema de estadiamento mais usado é o TNM, revisado em 2002 pela União Internacional Contra o Câncer (UICC) $)^{6,7}$. Nesta nova classificação, o tumor primário 
é estadiado de acordo com as características histológicas e com uma descrição mais precisa da estrutura anatômica envolvida, sendo esse último modelo de classificação mais completo que o modelo proposto por Jackson.

O sistema de estadiamento TNM oferece informações relacionadas ao tamanho do tumor, à quantidade e tamanho dos nódulos regionais acometidos e, finalmente, à presença de metástase à distância. As informações combinadas sobre tumor, nódulos linfáticos e metástase determinam 0 estadiamento, que é descrito em números romanos, variando de I a IV 6,7

\section{Prevenção}

A prevenção do câncer de pênis é de fundamental importância, pois a doença está relacionada a hábitos sexuais e de higiene precários. Sendo assim, é necessário intensificar as campanhas de prevenção, repassando à população o conhecimento sobre a associação dos maus hábitos de higiene e 0 efeito carcinogênico da fimose e da infecção pelo HPV na gênese do câncer de pênis.
Portanto, a imunização de adolescentes do sexo masculino é fundamental para interromper o ciclo de transmissão do $\mathrm{HPV}^{4}$. Surpreendentemente, apenas três pacientes foram diagnosticados com HIV/AIDS (vírus da imunodeficiência humana, síndrome da imunodeficiência adquirida) ${ }^{9}$.

Medidas importantes para a prevenção do Câncer de pênis são o autoexame, a inspeção e a limpeza diária com água e sabão. Campanhas de prevenção podem diagnosticar o câncer de pênis nos estágios iniciais, reduzir a incidência e a severidade da doença, e também proporcionar maiores chances de cura e melhora da sobrevida ${ }^{4,6}$.

A utilização do preservativo é imprescindível em qualquer relação sexual, já que a prática com diferentes parceiros sem o uso de camisinha aumenta o risco de desenvolver a doença. $O$ preservativo diminui a chance de contágio de doenças sexualmente transmissíveis como o HPV4. A cirurgia de fimose é outro fator de prevenção. A operação é simples e rápida e não necessita de internação.

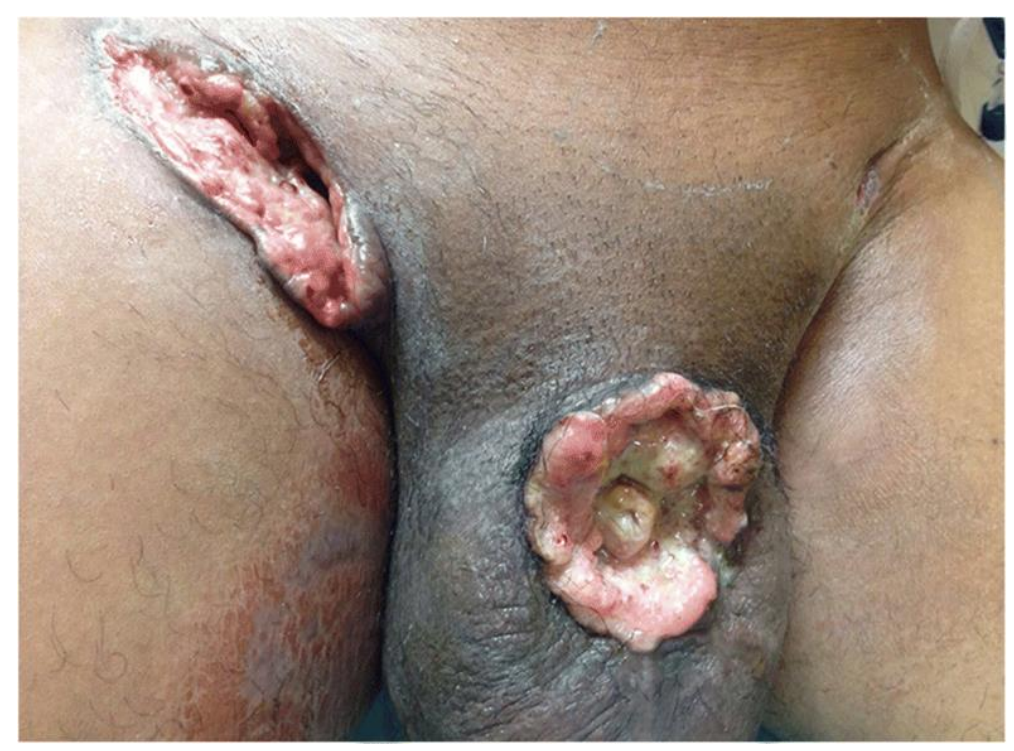

Figura 1. Paciente de 42 anos com doença avançada: auto-penectomia e fístula inguinal. ${ }^{9}$. 


\section{Considerações finais}

O câncer de pênis no Brasil atinge
indivíduos de baixa condição
socioeconômica e baixo grau de
escolaridade, localizados principalmente
no Norte e Nordeste. A presença de
fimose, higienização inadequada,
infecções virais e comportamento sexual
de risco constituem os principais fatores
para o desenvolvimento de câncer de
pênis. O estado do Maranhão tem ma maior
incidência de câncer de pênis no Brasil e
no mundo. Os tumores costumam estar
localmente avan çados no momento do diagnóstico e
há uma alta frequência entre os jovens. O
diagnóstico precoce é fundamental para
evitar o desenvolvimento da doença e a
amputação, acarretam
consequências físicas, sexuais e
psicológicas para o paciente. Diante de
um baixo nível socioeconômico entre os
pacientes acometidos, existe uma
dificuldade de conclusão do tratamento e
o acompanhamento adequado. Desta
forma é necessário que recursos
financeiros sejam destinados a atender,
por meio de campanhas educativas
preventivas, a população carente de
informações sobre esta patologia.

\section{Referências}

1. Costa S, Rodrigues R, Barbosa L, Silva J, de Caldas Brandão JO, de Medeiros CSQ. Câncer de pênis: epidemiologia e estratégias de prevenção. Caderno de Graduação-Ciências Biológicas e da Saúde-FACIPE. 2013;1(2):2333.

2. Pompeo ACL, Heyns CF, Abrams P (eds). Penile Cancer. Montreal: Société Internationale d'Urologie (SIU), 2009.

3. Barnholtz-Sloan JS, Maldonado JL, Pow-sang $\mathrm{J}$, Giuliano AR. Incidence trends in primary malignant penile cancer. Urologic Oncology 2007; 25(5): 361-67.

4. Instituto Nacional de Câncer José Alencar Gomes da Silva. Estimativa 2012: incidência de câncer no Brasil. Coordenação Geral de Ações Estratégicas, Coordenação de Prevenção e Vigilância. Rio de Janeiro: Inca; 2011. 118 p.

5. Brasil. Ministério da Saúde. Departamento de DST, AIDS e Hepatites Virais. Previna-se. Por que usar camisinha. Disponível em:< http://www.aids.gov.br/pagina/2010/42967> Acesso em: 29/05/2017.

6. Brasil. Instituto Nacional do Câncer 2017. Disponivel em: http://www1.inca.gov.br/. Acesso em: 22 de agosto de 2017.

7. Brasil. Ministério da Saúde. TNM: classificação de tumores malignos. 6. ed. Rio de Janeiro: INCA, 2004.

8. Pow-Sang MR, Benavente V, Pow-Sang JE, Morante C, Meza L, Baker M, et al. Cancer of the penis. Cancer control. 2002;9(4):305-14.

9. Coelho RWP, Pinho JD, Moreno JS, do Nascimento AMT, Larges JS, Calixto JRR, et al. Penile cancer in Maranhão, Northeast Brazil: the highest incidence globally? BMC urology. 2018;18(1):50. 